\title{
Computation of Eigenvalues of Singular Sturm-Liouville Systems
}

\author{
By D. O. Banks and G. J. Kurowski
}

1. Introduction. In recent papers, P. B. Bailey [2] and M. Godart [5] have used the Prüfer transformation to calculate the eigenvalues of nonsingular and some singular Sturm-Liouville boundary value problems. This method has the advantage over usual initial-value and algebraic methods (see e.g. [3], [4] and others) since the $n$th eigenvalue is obtained directly by prescribing $n$. The use of the Prüfer transformation for the computation of eigenvalues of nonsingular problems was also suggested by Kamke ([6, pp. 269-270]). A similar method was used by Milne [8].

In the present article, we establish the existence of a general class of singular problems which may be solved in a straightforward manner using the Prüfer transformation. For simplicity, in Section 2, we describe the method for nonsingular Sturm-Liouville problems. In Section 3, we show that this method is applicable to a large class of singular problems. Some examples are given in Section 4 . In Section 5 , we extend the class of singular problems to which this method is applicable by introducing a modified transformation.

2. Computation of Eigenvalues for Nonsingular Problems. We consider the problem of computing the eigenvalues for boundary-value problems associated with the Sturm-Liouville equation

$$
\left(p(x) u^{\prime}\right)^{\prime}+[\lambda \rho(x)-q(x)] u=0, \quad x \in(a, b),
$$

where the interval $[a, b]$ is finite. On the closed interval $[a, b]$, we take $p, p^{\prime}, \rho$ and $q$ to be real and continuous where $p$ and $\rho$ are positive. For this nonsingular problem, we consider boundary conditions of the form

$$
\begin{gathered}
u(a) \cos A-p(a) u^{\prime}(a) \sin A=0, \\
u(b) \cos B-p(b) u^{\prime}(b) \sin B=0 .
\end{gathered}
$$

Applying the Prüfer transformation, we introduce the new variables $r(x)$ and $\theta(x)$ defined by

$$
u(x)=r(x) \sin \theta(x), \quad p(x) u^{\prime}(x)=r(x) \cos \theta(x) .
$$

Under this transformation, we obtain the equation

$$
\theta^{\prime}=(1 / p(x)) \cos ^{2} \theta+[\lambda \rho(x)-q(x)] \sin ^{2} \theta
$$

with boundary conditions

$$
\begin{aligned}
& \theta(a)=A, \\
& \theta(b)=B+n \pi,
\end{aligned}
$$

Received July 17, 1967. 
where $-\pi / 2 \leqq A<\pi / 2,-\pi / 2 \leqq B<\pi / 2$, and $n$ is a nonnegative integer.

To find the $n$th eigenvalue of (1), (2), (3), we proceed as follows. For a given value of $\lambda$, we integrate (5) from $a$ to $(a+b) / 2$ using (6) as an initial condition to produce the solution $\theta_{a}(x, \lambda)$ for $x \in[a,(a+b) / 2]$. For this same value of $\lambda$ we integrate from $b$ back to $(a+b) / 2$ using (7) with a particular choice of $n$ as an initial condition to produce the solution $\theta_{b}(x, \lambda)$ for $x \in[(a+b) / 2, b]$. When $\lambda$ equals the $n$th eigenvalue $\lambda_{n}$

$$
E\left(\lambda_{n}\right)=\theta_{a}\left((a+b) / 2, \lambda_{n}\right)-\theta_{b}\left((a+b) / 2, \lambda_{n}\right)=0 .
$$

The determination of the $n$th eigenvalue is thus equivalent to solving the transcendental equation (8). Although Godart [5] has suggested this procedure in the case of problems which are singular at both $a$ and $b$, it is advantageous to always integrate from both $a$ and $b$ since the accumulation of rounding errors will be reduced.

Equation (8) can be solved by iteration. In [5], Godart applies the NewtonRaphson approximation method. That is, for an initial value $\lambda_{n}{ }^{\left({ }^{0}\right)}$ successive estimates of $\lambda_{n}$ are given by

$$
\lambda_{n}{ }^{(k+1)}=\lambda_{n}{ }^{(k)}-E\left(\lambda_{n}{ }^{(k)}\right) / E^{\prime}\left(\lambda_{n}{ }^{(k)}\right)
$$

where $E^{\prime}(\lambda)$ is obtained as follows. Defining

$$
\chi(x, \lambda)=\partial \theta(x, \lambda) / \partial \lambda,
$$

differentiation of (5) yields

$$
\chi^{\prime}=\rho \sin ^{2} \theta+\chi[\lambda \rho-q-1 / p] \sin 2 \theta .
$$

From (6) and (7), we obtain the conditions

$$
\chi(a, \lambda)=0 ; \quad \chi(b, \lambda)=0 .
$$

Thus for each iterative step in addition to solving (5) to obtain $E$, we must also solve (11) to obtain $E^{\prime}$.

3. Computation of Eigenvalues of Singular Problems. If (1) has regular singular points at $x=a$ or $x=b$, it would appear that a different technique of integration is required. We shall show, however, that for a wide class of such singular problems, this is not the case. As pointed out by Bailey [2], the asymptotic behavior of the solutions of (1) usually serves to determine the boundary condition for $\theta$ at the singular points. By studying this asymptotic behavior, we determine a class of singular points for which the method described in Section 2 can be applied directly.

We start by considering the normal form

$$
u^{\prime \prime}+p_{1}(x) u^{\prime}+p_{2}(x) u=0, \quad x \in(0, c),
$$

which we take to have a regular singular point at $x=0$ where

$$
p_{1}(x)=\alpha / x+S(x)
$$

and

$$
p_{2}(x)=Q(x) / x^{2}
$$

with $S$ and $Q$ analytic. We put (13) in self-adjoint form by multiplying by $\exp \int^{x} p_{1} d x$ 
$=x^{\alpha} P(x)$ where $P(x)=\exp \int^{x} S d t$. We obtain

$$
\left(x^{\alpha} P(x) u^{\prime}\right)^{\prime}+x^{\alpha-2} P(x) Q(x) u=0
$$

and note that $P(x)>0$ for $x \geqq 0$ and $Q(x)=x^{\gamma} R(x)$ where $R(0) \neq 0$ and $\gamma \geqq 0$.

The Prüfer transformation (4) applied to (14) yields

$$
\theta^{\prime}=x^{\alpha+\gamma-2} P(x) R(x) \sin ^{2} \theta+\left[x^{\alpha} P(x)\right]^{-1} \cos ^{2} \theta .
$$

To apply the method of Section 2 , it is necessary that $\theta^{\prime}$ have a finite value at $x=0$. Using (4) we may write (15) in the form

$$
r^{2}(x) \theta^{\prime}=x^{\alpha+\gamma-2} P R u^{2}+x^{\alpha} P u^{\prime 2}
$$

where

$$
r^{2}(x)=u^{2}+x^{2 \alpha} P^{2} u^{\prime 2} .
$$

For $\theta^{\prime}$ to be finite at $x=0$, solutions of (13) must be of the form $u(x)=x^{\beta} U(x)$ where $U(0) \neq 0$ and $U$ is analytic. It will be convenient to consider two cases.

Case I. $\beta>0$. Then $u^{\prime}(x)=x^{\beta-1} V(x)$ where $V(x)=\beta U+x U^{\prime}(x)$ is analytic and $V(0) \neq 0$. From (16) we then obtain

$$
\theta^{\prime}=\frac{x^{\gamma} P R U^{2}+P V^{2}}{x^{2-\alpha} U^{2}+x^{\alpha} P^{2} V^{2}} .
$$

The numerator of (18) is not zero at $x=0$. The denominator of (18) vanishes for $x=0$ if $0<\alpha<2$. Consequently, $\theta^{\prime}$ is not bounded near $x=0$ for this range of values of $\alpha$. However, for all other values of $\alpha, \theta^{\prime}$ is well defined. One notes that this behavior is independent of the value of $\gamma$, the order of the zero of $Q(x)$ at $x=0$.

Case II. $\beta=0$. In this case $r(x)$ does not vanish and remains finite if $\alpha \geqq 0$. Thus for $\alpha \geqq 0$ we need only consider the behavior of the first term on the right side of (16). Since $P R u^{2} \neq 0$, this term is finite if and only if

$$
\gamma \geqq 2-\alpha \text {. }
$$

Consequently, if the order of the zero of $Q(x)$ is not less than $2-\alpha, \theta^{\prime}$ is finite.

To complete the discussion of this case, we must consider negative values of $\alpha$. It follows in a similar manner that $\theta^{\prime}$ is finite when (19) holds and $u^{\prime}(0)=0$. When $u^{\prime}(0) \neq 0$ it is required that

$$
\gamma \geqq 2+\alpha .
$$

Our results are summarized in the following:

THEоREM 1. If there is a solution of the form $u(x)=x^{\beta} U(x)(U(0) \neq 0, \beta \geqq 0)$ of the equation

$$
\left(x^{\alpha} P(x) u^{\prime}\right)^{\prime}+x^{\alpha-2} P(x) Q(x) u=0
$$

where $P(x)>0$ and $Q(x)=x^{\gamma} R(x)(R(0) \neq 0)$, then $\theta^{\prime}$ is finite in a neighborhood of $x=0$ provided that one of the following conditions holds:

(i) $\beta>0$ and $\alpha \leqq 0$ or $\alpha \geqq 2$,

(ii) $\beta=0, \alpha \geqq 0$ and $\gamma \geqq 2-\alpha$,

(iii) $\beta=0, \alpha<0, u^{\prime}(0)=0$ and $\gamma \geqq 2-\alpha$,

(iv) $\beta=0, \alpha<0, u^{\prime}(0) \neq 0$ and $\gamma \geqq 2+\alpha$. 
We remark that if $\theta^{\prime}$ is computable at a singular point under the conditions of this theorem then $\chi^{\prime}$ of (11) will also be computable. This may be seen by noting that

$$
r^{2} \sin 2 \theta=r^{2} 2 \sin \theta \cos \theta=2 p u u^{\prime} .
$$

We note that our theorem does not apply to Bessel's equation of order $\nu>0$,

$$
\left(x u^{\prime}\right)^{\prime}+\left[\lambda x-\nu^{2} / x\right] u=0,
$$

since $\alpha=1$ and $\beta>0$. In [2], Bailey solves Bessel's equation with $\nu=1 / 4$ using the Prüfer transformation. A modification of the analysis used in proving the above theorem shows that the method can be applied to (14) whenever $\alpha=1$ and $\beta>0$. To see this we again consider a solution of the form $u(x)=x^{\beta} U(x)$. This implies that $\gamma=0$ and $\beta=(-R(0))^{1 / 2}$. From (16) and the fact that $V(x)=\beta U+x U^{\prime}(x)$, we obtain

$$
\left[U^{2}+x^{2} P^{2} V^{2}\right] \theta^{\prime}=x^{-1} P(x)\left[R(x)+\beta^{2}\right] U^{2}+2 \beta P(x) U U^{\prime}+x P(x) U^{\prime 2} .
$$

But $\beta^{2}=-R(0)$, consequently the first term on the right is finite and $\theta^{\prime}$ is computable in a neighborhood of the origin.

4. Some Examples. Before presenting examples of the use of the above theorem, we shall briefly describe the procedure used for the numerical solution of each of the example problems. The differential equations were first written as a system of firstorder equations. The classic Runge-Kutta fourth-order method (see Collatz [3]) was used to obtain starting values. The remainder of the computation was carried out using a predictor-corrector method obtained from the Adams extrapolation and interpolation methods described by Collatz [3] by retaining differences through the third order. The corrector was applied once per computational step, an estimate of the local truncation error was made at each step to ensure that this error did not exceed a predetermined tolerance.

In [4], Fox considers the problem of an oscillating elliptically shaped narrow lake. This leads to the solution of the boundary value problem

$$
\begin{aligned}
u^{\prime \prime}+x u^{\prime} /\left(1-x^{2}\right)+\lambda u & =0, \quad x \in(-1,1) \\
u( \pm 1) & =0 .
\end{aligned}
$$

For this problem we have $\alpha=-1 / 2, \beta=3 / 2$ and, consequently, it falls under case (i) of the above theorem. The following table gives computed values for $\lambda_{i}, i=$ $1,2,3$ of (21) for three step sizes $h$ in the integration scheme.

TABLE I

\begin{tabular}{c|c|c|c}
\hline$h$ & $\lambda_{1}$ & $\lambda_{2}$ & $\lambda_{3}$ \\
\hline 0.020 & 3.56058 & 12.1619 & 25.6795 \\
0.010 & 3.55974 & 12.1583 & 25.7090 \\
0.005 & 3.55944 & 12.1570 & 25.7051 \\
\hline
\end{tabular}


The values of $\lambda_{1}$ in the table compare favorably with the value $\nu=(0.04) \lambda_{1}=$ 0.1423 given by Fox.

Bessel's equation of order zero has $\alpha=1, \beta=0$ and is an example of case (ii). We have

$$
\left(x u^{\prime}\right)^{\prime}+\lambda x u=0, u(0) \text { finite }, u(1)=0 .
$$

The results of the computation are summarized in Table II.

TABLE II

\begin{tabular}{c|c|c|c}
\hline$h$ & $\lambda_{1}$ & $\lambda_{2}$ & $\lambda_{3}$ \\
\hline 0.0100 & 5.78318 & 30.4713 & 74.8598 \\
0.0050 & 5.78318 & 30.4712 & 74.8873 \\
0.0025 & 5.78318 & 30.4712 & 74.8867 \\
\hline Exact $\lambda_{i}$ & 5.783186 & 30.47126 & 74.88701 \\
\hline
\end{tabular}

Bailey [2] considers the equation

$$
\left(\left(1-x^{2}\right) u^{\prime}\right)^{\prime}+\left[\lambda x^{2}-(5 x+22.55316)\right] u=0, \quad x \in(-1,1),
$$

associated with the study of the energy levels of the hydrogen-helium molecular ion. Both singular points of this example fall under case (ii). Thus we may integrate (5) from both ends and avoid the computational difficulties of integrating into a singular point. Godart [5] did this to find eigenvalues of Legendre's equation. Using this method with an integration step of 0.005 , we obtained $\lambda_{1}=27.50301$, which agrees favorably with previous results.

As would be expected, the convergence of the Newton-Raphson iteration depends on the initial guess $\lambda_{n}{ }^{(0)}$. It is possible to obtain estimates of $\lambda_{n}$ using asymptotic formulae or other methods as given in [1], [3], and [7]. In particular, we note that the initial choice of $\lambda_{1}$ for equation (23) is especially critical.

Finally, we note that we have been unable to find specific examples in mathematical physics which fall under cases (iii) and (iv) of the theorem.

5. A Modified Transformation. By the analysis which leads to Theorem 1 , one sees that the Prüfer transformation cannot be used when $\alpha \in(0,1)$ or $\alpha \in(1,2)$ and $\beta>0$. One might hope that the alternative Prüfer transformation

$$
u(x)=r(x) \cos \psi(x), \quad p(x) u^{\prime}(x)=r(x) \sin \psi(x)
$$

could be used to solve such singular problems. However, the use of (24) in the analysis of $\psi^{\prime}$ yields (16) with $-\psi^{\prime}$ replacing $\theta^{\prime}$. Hence, no advantage is gained using this transformation.

To compute the eigenvalues for such values of $\alpha$, we define a new function $\phi$ by the equation

$$
\phi=\left(\theta-\theta_{0}\right)^{n+1}
$$

where $\theta_{0}=\theta(0)$ and $n$ is a parameter to be determined. Equation (5) then yields 


$$
\phi^{\prime}=(n+1) \phi^{n /(n+1)}\left[(1 / p) \cos ^{2} \theta+(\lambda \rho-q) \sin ^{2} \theta\right]
$$

where

$$
\theta=\phi^{1 /(n+1)}+\theta_{0} .
$$

We note that $\phi$ will be a solution of $(26)$ with $\phi(0)=0$. With this initial condition there is no unique solution of (26) since $\phi \equiv 0$ together with the nonzero solution obtained from (1) satisfy this equation. We can find a unique nonzero solution by choosing $\phi^{\prime}(0)$ to be the value obtained from the investigation of the asymptotic value of $\phi^{\prime}(x)$ as $x \rightarrow 0$. We proceed as follows.

Again assuming there is a solution of the form $u(x)=x^{\beta} U(x), \beta>0$, we see that

$$
\begin{aligned}
\theta_{0}=\lim _{x \rightarrow 0} \tan ^{-1} \frac{u}{p(x) u^{\prime}} & =0, \quad \alpha<1, \\
& =-\pi / 2, \quad \alpha>1 .
\end{aligned}
$$

Also in the neighborhood of the origin, we have

$$
\begin{aligned}
\theta(x)=\tan ^{-1} \frac{u}{p u^{\prime}} & =\frac{U(x)}{x^{\alpha-1} P(x) V(x)}+O\left[x^{3-3 \alpha}\right], \quad \alpha<1, \\
& =-\pi / 2-\frac{x^{\alpha-1} P(x) V(x)}{U(x)}+O\left[x^{3 \alpha-3}\right], \quad \alpha>1,
\end{aligned}
$$

where $p(x)=x^{\alpha} P(x)$ and $V(x)=\beta U(x)+x U^{\prime}(x)$ as before. For $\alpha \in(0,1)$.

$$
\phi^{\prime}=(n+1)\left[\frac{U}{x^{\alpha-1} P V}+O\left(x^{3-3 \alpha}\right)\right]^{n} \frac{x^{\gamma-\alpha} P R U^{2}+P V^{2} x^{-\alpha}}{x^{2-2 \alpha} U^{2}+P^{2} V^{2}}
$$

is finite and nonzero at $x=0$ when $n=\alpha /(1-\alpha)$.

It follows from (29) that, for this value of $n$,

$$
\begin{aligned}
\lim _{x \rightarrow 0} \phi^{\prime}(x) & =(n+1)[\beta P(0)]^{-n}\left(R(0)+\beta^{2}\right) /\left(P(0) \beta^{2}\right), \quad \gamma=0, \\
& =(n+1) \beta^{-n}[P(0)]^{-n-1}, \quad \gamma>0 .
\end{aligned}
$$

For $\alpha \in(1,2)$,

$$
\phi^{\prime}=(n+1)\left[\frac{-x^{\alpha-1} P V}{U}+O\left(x^{3 \alpha-3}\right)\right]^{n} \frac{x^{\gamma+\alpha-2} P R U^{2}+x^{\alpha-2} P V^{2}}{U^{2}+x^{2 \alpha-2} P^{2} V^{2}}
$$

is finite and nonzero at $x=0$ when $n=(2-\alpha) /(\alpha-1)$. As above, it follows from (31) that, for this value of $n$,

$$
\begin{aligned}
\lim _{x \rightarrow 0} \phi^{\prime}(x) & =(n+1)(-\beta)^{n}[P(0)]^{n+1}\left[R(0)+\beta^{2}\right], \quad \gamma=0, \\
& =(n+1)(-\beta)^{n+2}[P(0)]^{n+1}, \quad \gamma>0 .
\end{aligned}
$$

These results are summarized in the following:

Theorem 2. If there is a solution of (14) with $\alpha \in(0,1)$ or $\alpha \in(1,2)$ of the form $u(x)=x^{\beta} U(x), \beta>0$ and $U(0) \neq 0$, where $P(x)>0$ and $Q(x)=x^{\gamma} R(x),(R(0) \neq 0)$, then $\phi^{\prime}(0)$ is finite, nonzero and is given by (30) for $\alpha \in(0,1)$ with $n=\alpha /(1-\alpha)$ and by (32) for $\alpha \in(1,2)$ with $n=(2-\alpha) /(\alpha-1) . \theta(x)$ may then be found from (26) and (27). 
Theorem 2 may be used to compute the eigenvalues of such singular problems in the following way. We find $\phi(x, \lambda)$ by integrating (26) and subsequently $\theta(x, \lambda)$ from (27) for a choice of $\lambda$. As before, with this function $\theta$, we integrate (11) to get $\chi(x, \lambda)$ and use (9) to obtain successive approximations for the eigenvalue.

As an example, we consider

$$
\left(x^{1 / 2} u^{\prime}\right)^{\prime}+\lambda u=0, \quad u(0) \text { finite }, \quad u(1)=0 .
$$

The parameters of this example are $\alpha=1 / 2, \beta=1 / 2, \gamma=3 / 2$. Thus $n=1$ and $\phi^{\prime}(0)=4$. The results of this computation are given in the following table. The first three eigenvalues are calculated using step sizes $h=1 / 128,1 / 256,1 / 512$, together with the exact values [6].

TABLE III

\begin{tabular}{c|c|c|c}
\hline$h$ & $\lambda_{1}$ & $\lambda_{2}$ & $\lambda_{3}$ \\
\hline $1 / 128$ & 4.73889 & 20.4710 & 47.3090 \\
$1 / 256$ & 4.73890 & 20.4714 & 47.3046 \\
$1 / 512$ & 4.73903 & 20.4715 & 47.3047 \\
\hline Exact $\lambda_{i}$ & 4.73907 & 20.4716 & 47.3052 \\
\hline
\end{tabular}

University of California

Davis, California

1. D. BANKs, "Upper bounds for the eigenvalues of some vibrating systems," Pacific J. Math., v. 11, 1961, pp. 1183-1203. MR 26 \#2681.

2. P. B. BAILEY, "Sturm-Liouville eigenvalues via a phase function," SI.AM J. Appl. MIath.,

v. 14, 1966, pp. 242-249.

3. L. Collatz, The Numerical Treatment of Ordinary Differential Equations, 3rd ed., SpringerVerlag, New York, 1966.

4. L. Fox, The Numerical Solution of Two-point Boundary Problems in Ordinary Differential Equations, Oxford Univ. Press, New York, 1957. MR 21 \#972.

5. M. GodarT, "An iterative method for the solution of eigenvalue problems," Math. Comp., v. 20, 1966, pp. 399-406. MR $34 \# 3775$.

6. E. KAMKE, Differentialgleichungen. Lösungsmethoden und Lösungen, Band 1, Chelsea, New York, 1959. MR $21 \# 5036$.

7. M. G. KREIN, "On certain problems on the maximum and minimum of characteristic values and on Lyapunov zones of stability," Amer. Math. Soc. Transl. (2), v. 1, 1955, pp. 163-187. MR 17, 484.

8. W. E. Milne, "On the numerical solution of a boundary value problem," Amer. Math. Monthly, v. 38, 1931, pp. 14-17. 